\title{
Zwei Interpretationen zum Hippokratischen Eid
}

\author{
Von Fridolf Kudlien
}

Den beiden folgenden Beiträgen ist gemeinsam, daß sie zwei Kerngelübde des «Eides» - das eine so umstritten, daß man es schließlich aus dem Text entfernen wollte - aus berufsethischen Grundvorstellungen althippokratischer Ärzte heraus erklären wollen. Sie wenden sich also dagegen, dem «Eid» den Charakter einer Exklusivität - sei es einer pythagoreischen (Edelstein), sei es sonst irgendeiner «absoluten Ausnahmestellung» (J.H. Wolf ${ }^{1}$ ) - zu geben. Insofern soll uns der «Eid» durchaus wieder zu einem hippokratischen Dokument werden. Ob er dennoch, und zwar wegen seines berufsethischen Rigorismus, in der Antike das «Dokument einer Minderheit» war (um eine früher benutzte Formel aufzugreifen), ist eine Frage, die berechtigt bleibt (s. Abschnitt 2).

\section{Wie religiös ist der «Eid»? ${ }^{2}$}

Ein Eid, Symbol einer «unlösbaren Verkettung von Religion, Moral und Organisation der Gesellschaft überhaupt» (Burkert), ist im alten Griechenland (wie in anderen alten Gesellschaften) ursprünglich ein religiöser Akt, zu dem ein Ritual (Eidopfer) und gegebenenfalls, als Ort der Eidesleistung, ein Heiligtum gehören ${ }^{3}$.

Beim Hippokratischen Eid ist nichts von Opfer und Heiligtum wahrzunehmen. Bemerkenswert ist aber vor allem auch, daß der ursprüngliche Charakter des Eides als "Selbstverfluchung» beziehungsweise «Weihung der eigenen Person an die Gottheit ${ }^{4}$ sowie der höchst konkrete Aspekt der Vernichtung für den Eidbrüchigen, wie er typisch ist für den echt religiösen Eid ${ }^{5}$, bei Hippokrates ganz abgeblaßt, profaniert ist («wenn ich aber verstoße und meineidig werde, das Gegenteil dessen » - das heißt Ruhmlosigkeit).

Wir wollen also für unser Thema nicht von der Nennung der Götter am Anfang ausgehen - sie wird hier wohl als formelhaft, zwar typisch für einen Eid, aber nicht Zeichen einer spezifischen Religiosität, zu nehmen sein -, sondern von dem

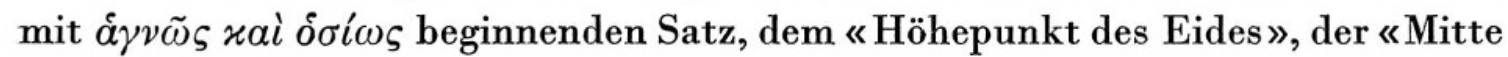
der Pflichtenlehre». ${ }^{6}$

Die genannten griechischen Worte werden in den bekanntesten Übersetzungen moderner Altertumswissenschaftler einhellig aufgefaßt: $\alpha \hat{\gamma} v \tilde{\omega} \varsigma$ «heilig» (Müri,

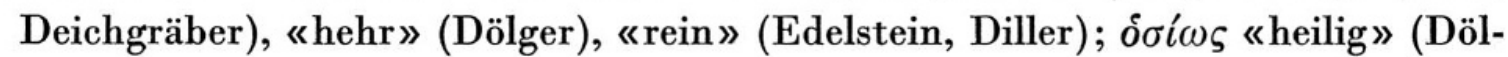
ger, Edelstein), «fromm» (Diller), «rein» (Müri, Deichgräber). Mit dieser Übersetzung - und allein mit ihr - kommt ein unverkennbar hochreligiöser Ton in den «Eid». ${ }^{7}$ Hohe Religiosität setzen auch die genannten Wissenschaftler, unter 
Berufung auf die beiden in Frage stehenden Worte, als selbstverständlich voraus ${ }^{8}$. So wäre denn der «Eid» doch ein Dokument hoher ärztlicher Religiosität und spezifisch religiöser Berufsmoral, die den Schwörenden zu einem «life almost saintly» verpflichtete ${ }^{9}$ ? Sollte etwa $\hat{\alpha} \gamma v$ ó $_{\zeta}$ hier den gleichen Beiklang haben wie $\dot{\varepsilon} v \alpha \gamma \eta$ ' in echt religiösen Eiden, wo letzteres «geradezu den Begriff der ... Heiligkeit einzuschließen scheint ${ }^{10}$ ?

Alle, die dieser Auffassung zuneigen ${ }^{11}$, verfahren, fürchte ich, voreilig und kurzschlüssig: Gewiß können áyvó stehen und haben dann die in den obigen Übersetzungen anklingende religiöse Bedeutung. Sie kommen aber auch in eindeutig profanen Kontexten vor und haben dann eine moralische, aber nicht spezifisch religiöse Bedeutung. Bisher scheint nur Bolkestein ${ }^{12}$ aufgrund philologischer Beweisführung (jedenfalls für ö $\sigma \circ)_{\text {) }}$ eine religiöse Bedeutung der beiden Adjektive im «Eid» abgelehnt und dafür eine profan-moralische (s.u.) vorgeschlagen zu haben. Dem ist Dölger nachträglich gefolgt ${ }^{13}$ (ihm kam es, wie er nun andeutete, nicht so sehr auf eine spezifisch religiöse, sondern auf Herausarbeitung einer ethischen Gesinnung und Motivation im «Eid» an).

Im folgenden wollen wir die profan-moralische Bedeutung beider Worte nochmals deutlich machen und dann diskutieren, ob nicht eher diese im «Eid» vorauszusetzen ist (womit der Hauptanhaltspunkt, den «Eid» als hochreligiöses Dokument aufzufassen, entfiele).

Von seinen indo-europäischen Ursprüngen her ist $\alpha$ $\gamma v o ́ \varsigma$, nach Benveniste ${ }^{14}$, zwar zunächst im religiösen Bereich anzutreffen, aber zu unterscheiden von $\ddot{\alpha} \gamma l o \varsigma$ («sacred») wegen seiner Eignung zu «nicht sehr präzisem Gebrauch»15 (das heißt für uns, zu weiter Anwendung). In ó $\gamma v o ́ s ~ s t e c k t$, wie in seinen indoeuropäischen Verwandten, "the idea of rigid conformity to a norm"16: Wer diese Norm erfüllt, ist «sauber», zunächst rituell, dann aber, wie nach der «unpräzisen» Natur des Wortes zu erwarten, auch in profan-moralischer Beziehung. Dieser «Ethisierung» des Wortes ist Williger nachgegangen ${ }^{17}$. In der Tat finden wir schon in Pindars Wettkampfdichtung (Olympien 3,21) die å $\gamma v \dot{\alpha} \varkappa \varrho i ́ \sigma \iota$, den «lauteren» (Wolde) oder, noch treffender, «sauber-unparteiischen Schiedsspruch » («gerecht» übersetzt hier Williger ${ }^{18}$ ). Bei anderen Autoren der klassischen Zeit steht das Wort neben $\delta$ ixalos «gerecht» und bedeutet dann etwa «frei von Charakterfehlern ${ }^{19}{ }^{19}$ Besonders zu beachten ist das Vorkommen in Inschriften, wo Amtsführung und Charakter eines Funktionärs im Profanbereich gelobt werden. Auch dafür gibt Williger Belege ${ }^{20}$. Das für uns Wichtigste: Hier bezeichnet

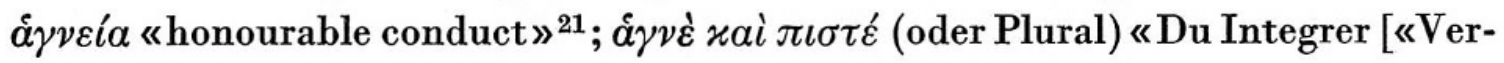
ehrter» übersetzt, weniger glücklich, Preisigke] und Vertrauenswürdiger» ist eine 
für Akklamationen von profanen Amtsträgern belegte Formel ${ }^{22}$; im gleichen Zu-

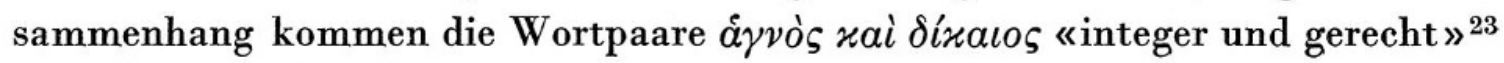
und $\hat{\alpha} \gamma \nu \tilde{\omega} \varsigma x \alpha i{ }_{\alpha} \mu \varepsilon \dot{\varepsilon} \mu \tau \tau \omega \varsigma$ «integer und untadlig» ${ }^{24}$ vor. Wenn ein scharfsin-

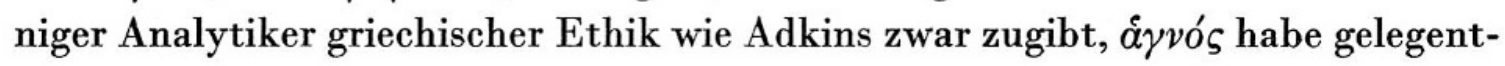
lich einen «strongly moral» Sinn, aber dennoch behauptet, es sei « of no use in moral contexts», da es nicht «purely moral», seine «logic» nicht die von «moral words» sei $^{25}$, dann übersieht er die Gesamtbezeugung des Wortes und gibt dessen religiösem Gebrauch zu einseitiges Gewicht - griechische «Vulgärmoral» war im profan-moralischen Gebrauch von å $\gamma v o ́ \varsigma$ offenbar frei von solchen Skrupeln.

Für ő $\sigma \iota \varsigma$ ist Ähnliches hervorzuheben: Die «paradoxe» Tatsache (Benveniste), daß es von Anfang an dienen konnte zur Bezeichnung von "what is sacred as [to] what is profane», erklärt sich nach Benveniste ${ }^{26}$ dadurch, daß "the term is applied to what is prescribed and permitted by divine law, but with reference to human relations». ${ }^{27}$ Das heißt, daß «the duties called 'hosia'... are duties towards men $»{ }^{28}$ Darin unterscheidet sich nach Benveniste ö $\sigma \circ \varsigma$ von

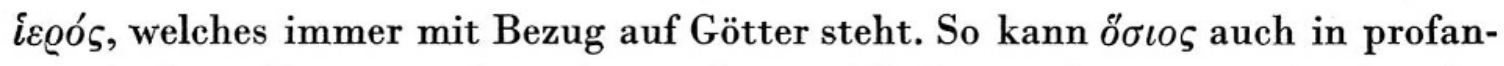
moralischem Kontext gebraucht werden und bedeutet dort etwa «legal» oder

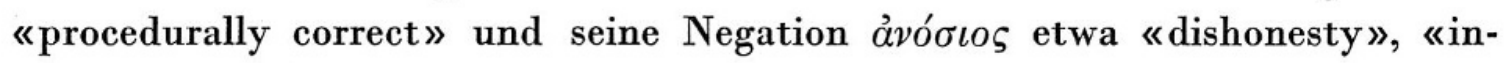
gratitude», «objectionable character», «political blackmail», «unpatriotic argument $2 .{ }^{29}$ Auch ist wieder das Vorkommen in inschriftlichen profanen Ehrungen bemerkenswert: Einem Amtsträger wird bescheinigt, er habe «ihm Anvertrautes

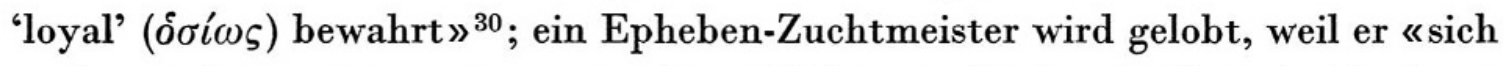
auch um das meiste andere mit aller Pflichttreue/Rechtschaffenheit (óøó $\eta ऽ \varsigma)$

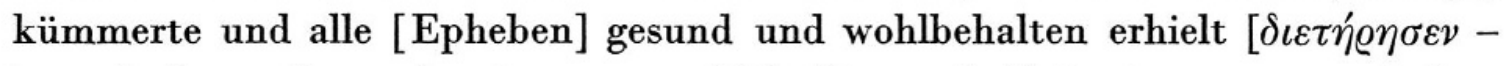
man denke an $\delta \iota \alpha \tau \eta \varrho \eta ́ \sigma \omega$ in unserer «Eid»-Passage] ${ }^{31}$; in einer pergamenischen

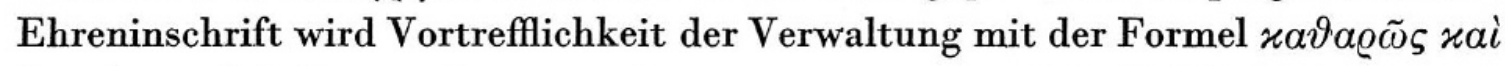

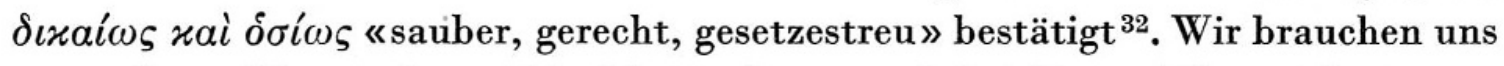
unter diesen Umständen wohl nicht zu fragen, «ob bei diesem Wort nicht immer

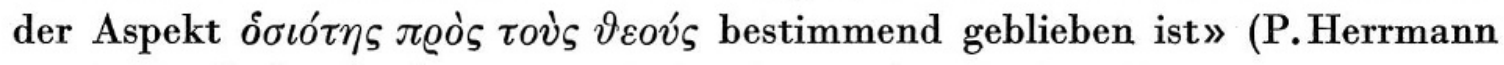
brieflich): So häufig dieser spezifisch religiöse Aspekt des Wortes auch bezeugt ist - seine profan-moralische Bezeugung reicht daneben aus, um eine solche

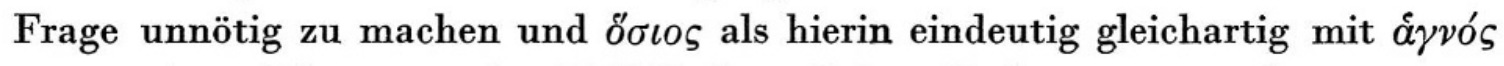
zu erweisen. Wenn van der Valk ${ }^{33}$ die religiöse Bedeutung von ö $\sigma \iota$ s primär betont, dann gilt für ihn die gleiche Kritik wie für Adkins: er übersieht die profan-moralische Bezeugung und damit die Gesamtbezeugung des Wortes.

Dieser philologische Befund muß ernstgenommen werden: Die Frage ist nun unabweisbar, ob im «Eid» eine spezifisch religiöse Bedeutung unserer beiden Worte angenommen werden $m u \beta$, oder ob nicht gerade in einer berufsethischen 
Ärzte-Verpflichtung der profan-moralische Sinn viel näherläge. Daß beide Worte hier Moralisches meinen, ist ja klar. Nur müssen sie eben deshalb nicht spezifisch Religiöses (generell Frömmigkeit, speziell rituelle Reinheit) meinen. Es gibt eine griechische "popular morality», die nicht nur unabhängig von philosophischer Ethik ist ${ }^{34}$, sondern auch derart in eine «identification of the patriotic, the lawabiding and the pious» mündete, daß eben deshalb bei Worten wie unseren Adjektiven, die ebenso der Religion verbunden wie der Profanierung fähig waren, «sometimes it is not easy to make any clear connection with religion». ${ }^{35}$

Wer nun immer noch, unter Berufung auf unsere Adjektive, eine spezifische Religiosität des «Eides» behauptet, hat die Beweislast zu tragen. Das Beibringen bloßer Ähnlichkeiten schafft keinen Beweis: Wenn die Satzung eines lydischen Privatkultes (frühes 1.Jahrhundert v. Chr.) dem das «Haus» Betretenden vorschreibt, er dürfe kein Gift und kein Abtreibungsmittel verabreichen und müsse sexuell sauber bleiben (das heißt, nur mit dem Ehepartner verkehren, keine Sklaven, Unmündigen, Geschlechtsgenossen sexuell mißbrauchen) ${ }^{36}$, dann ist das zwar frappant ähnlich einigem im «Eid». Aber beide Dokumente gehören ja in ganz verschiedene Kontexte: Im Kultgesetz ist das nur von «Reinen» zu betretende «Haus» ein Kulthaus, und seine Gebote und Verbote sind motiviert durch Rücksicht auf Götter und Umgang mit diesen. Der Inhalt der Kultgebote jedoch ist, an sich genommen, nichts als gewöhnliche «gute griechische Moral» (Nilsson). Diese hat der «Eid»-Verfasser, neben fachspezifischeren hippokratischen Moralvorstellungen, seiner Berufsethik zugrunde gelegt (er ist dabei allerdings so strikt, daß er bekanntlich in einigen Forderungen von Gepflogenheiten anderer Hippokratiker abweicht; s. auch Abschnitt 2). Seine Motive dabei aber sind ausdrücklich, und ausschließlich, beruflich-ärztliche: Rücksicht auf den «Nutzen der Patienten», Wunsch nach «Ruhm bei allen Menschen für alle Zeit»

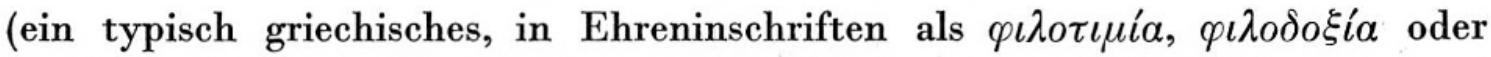
ähnlich bezeichnetes Motiv). Von religiöser Motivation und Rücksicht (etwa auf «Befleckung» als «größten Schaden», Deichgräber) ist hier nichts zu sehen. Dasselbe gilt für ein anderes, unsere Adjektive verwendendes Zeugnis, den Spruch, der über dem Eingang des Asklepiostempels in Epidauros stand ${ }^{37}$ : «ơ $\gamma$ vós muß der sein, der in den ... Tempel geht; $\tilde{\alpha} \gamma \nu \varepsilon i \eta \eta$ heißt, ö $\sigma \iota \alpha$ zu denken». Die beiden Worte haben hier natürlich religiösen Sinn. Es ist ja ein Tempel und ein (übrigens nicht nur als Heiler fungierender) Gott, die entsprechende Gesinnung und entsprechendes Verhalten fordern. Daß Asklepios daneben auch Patron der Ärzte ist, darf nicht dazu verleiten, zwei wesensmäßig ganz verschiedene Sphären wie Tempel/Kult und reguläre ärztliche Praxis aufeinander zu beziehen.

So plädiere ich dafür, unseren «Eid»-Passus mit Bolkestein («rein en recht- 
schapen») zu übersetzen als «integer und rechtschaffen/gesetzestreu werde ich mein Leben und mein Fach $\left(\tau \varepsilon^{\prime} \chi \nu \eta\right)$ bewahren». Der Mediziner O. Körner hätte demnach mit seinem «ohne Fehl und unbescholten» Richtiges erfaßt. Ich meine nunmehr, daß die religiöse Interpretation von $\alpha \gamma \nu \tilde{\omega} \varsigma$ $x i$ ó $\sigma i \omega \varsigma \mathrm{zu}$ den anderswo ${ }^{38}$ diskutierten «overstatements » zu rechnen ist; daß die profan-moralische Deutung dieser Worte einen weiteren Beleg für die früher von mir untersuchten Beziehungen zwischen ärztlicher und «popular» Ethik in Griechenland liefert; daß weder die Gebote und Verbote des «Eides» (auch nicht sein Schweigegebot, dessen Aus-

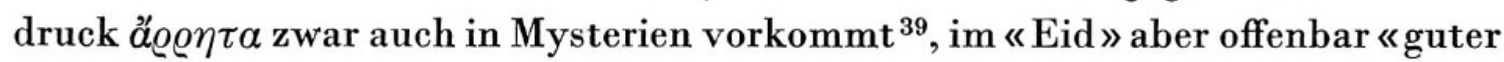
griechischer Moral» entspricht ${ }^{40}$ ) noch sein «covenant» ${ }^{41}$ irgendeine spezifische Religiosität (über die übliche offizielle hinaus) bezeugen.

\section{Warum keine Blasensteinoperation?}

Das Gelübde $o \dot{v} \tau \varepsilon \mu \dot{\varepsilon} \omega \delta \dot{\varepsilon} o v ̉ \delta \dot{\varepsilon} \mu \dot{\eta} \nu \lambda \iota \vartheta \hat{\omega} \nu \tau \alpha \varsigma$ hat besonders viel Befremden hervorgerufen ${ }^{42}$. Es enthält ein sprachliches und ein sachliches Problem, untrennbar miteinander verknüpft, die sich mit Diller ${ }^{43}$ so formulieren lassen: «(Dieser Satz) wäre weniger überraschend, wenn er nur eine Ablehnung der Operation des Blasensteins ... enthalten würde. Doch lassen sich diese Deutungen mit dem griechischen Text weniger leicht vereinbaren ». Zu denen, die dieses Gelübde nur auf Blasensteinoperation, nicht auf chirurgisches Eingreifen überhaupt, beziehen,

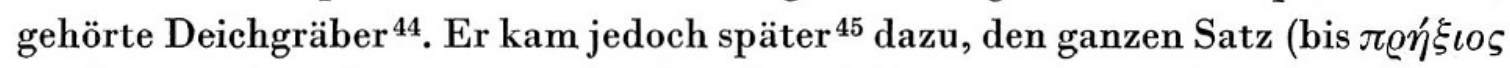
$\tau \tilde{\eta} \sigma \delta \varepsilon$ ) aus dem Text auszuschließen («es fehlt uns... jede Sicherheit, diesen Passus im Zusammenhang des Eides zu erklären»). In dieser Skepsis war Jones ${ }^{46}$ vorangegangen, der hier «an addition of late but uncertain date» vermutete.

Vor der sprachlichen Erklärung der entscheidenden Partikeln $o v \dot{\varepsilon} \delta \dot{\varepsilon} \eta^{\prime} v$ wollen wir das Sachliche von einem, wie uns scheint, bisher vernachlässigten Gesichtspunkt aus diskutieren. Bezöge sich unser Gelübde auf generellen Operationsverzicht, dann wäre es in der Tat überraschend und widerspräche antiker Praxis kraß. Beziehen wir es aber zunächst vor allem auf Blasensteinoperation, dann fügt es sich voll in den Rahmen althippokratischer Anschauungen. Einschneiden in die Blase galt hier als schädlich/gefährlich: «Eine durchtrennte Harnblase wächst nicht zusammen» (Morb. I 8, Ende); «man stirbt, wenn man verletzt wird an ... der Harnblase » (ebd. I 3; vgl. Coac. 499). Die dem zugrundeliegendeVor-

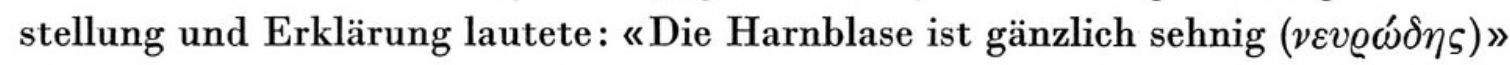
(Anatom.1). Wenn man eine Sehne oder etwas Sehniges durchtrennt, dann «wächst es nicht zusammen » (Morb. I 4 und 8; Aphorism. VI 19 und VII 28; Coac. 494) und verursacht «Krampf» (Morb.I 4). Man soll da also keinesfalls einschneiden (Morb.I 10). Berufsethisch bemerkenswert ist, daß Morb.I 8 betont, ein Arzt 
werde unter anderem wegen derartigem beschuldigt. Daher der Hinweis (ebd.), daß es zu den «guten» therapeutischen Maßnahmen gehöre, bei Blasensteinkranken auf medikamentösem Wege Abgang zu schaffen.

Es liegt auf der Hand, daß der «Eid»-Verfasser, der so sehr auf dem (in althippokratischer Berufsethik obenan stehenden ${ }^{47}$ ) Gebot «nützen oder (doch) nicht schaden» beharrt (vgl. das zweimalige $\dot{\varepsilon} \pi \dot{\omega} \varphi \varepsilon \lambda \varepsilon i \eta \eta \eta ~ \varkappa a \mu \nu o ́ v \tau \omega \nu$ sowie den Hinweis auf $\delta \eta^{\prime} \lambda \eta \sigma \iota \varsigma$ und $\left.\dot{\alpha} \delta \iota x i \eta\right)$, unter diesen Umständen wenigstens die Blasensteinoperation ablehnen mußte. Läßt sich das auch vom Sprachlichen her untermauern?

$o v ̉ \delta \dot{\varepsilon}$ nimmt man an unserer «Eid»-Stelle gewöhnlich als verstärkende Negation ${ }^{48}$. So kann es gebraucht werden. Falsch ist aber, wenn man nur an diese Möglichkeit denkt und behauptet «ov̉ঠé always means 'not even'».49 Auch bei $\mu \eta \dot{v}$ denkt man hier nur an die beteuernde ${ }^{50}$ oder einen Extremfall betonende («sogar»; in Verneinungen «[nicht] einmal») Bedeutung, während auch dies nur eine der Übersetzungsmöglichkeiten ist. Selbst Denniston, hervorragender Kenner des griechischen Partikelgebrauchs, versteht ov̉ $\delta \dot{\varepsilon} \mu \eta \dot{\eta} v$ im «Eid» als «not even », fügt aber hinzu «but the whole sentence is much disputed» ${ }^{51}$ (dazu paßt Jones' ${ }^{52}$ Bemerkung: «the $\mu \eta v^{\prime} \ldots$ will strike scholars as strange»).

Denniston oder andere Darstellungen griechischen Partikelgebrauchs hätten freilich lehren können, daß es weitere Übersetzungsmöglichkeiten von ov̉dé und $\mu \eta^{\prime}$ gibt, die die obige sachliche Deutung sprachlich unterstützen: ov̉dé kann «responsive» Verneinung («simply adding a negative idea, usually to a negative idea either expressed or implied»53) oder, noch passender für unsere Stelle, «duplication of negative» («ov̉ is followed by responsive ov̉oć» ${ }^{54}$ ) sein. Und für $\mu \eta^{\prime}$ paßt hier besonders ein «progressiver» Gebrauch («marking the transition from major to minor premise ${ }^{55}$ ). $o v ่ \tau \varepsilon \mu \varepsilon ́ \omega$ «ich werde nicht schneiden» wäre

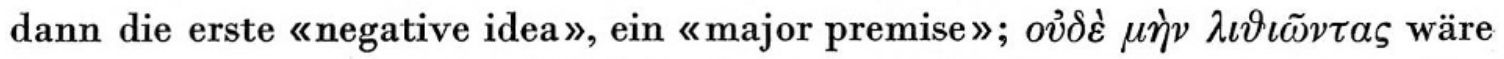
das «responsive» negative Gelübde, das entsprechende «minor premise». Der ganze Satz wäre demnach zu übersetzen: «ich werde nicht schneiden, jedenfalls. Steinleidende nicht».

Kein sprachliches, aber ein sachliches Problem bietet der Ergänzungssatz.

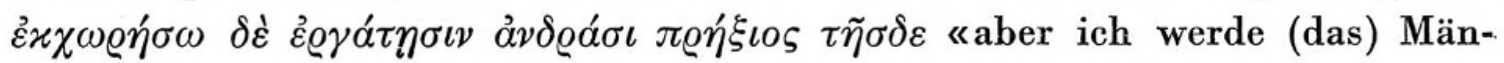
nern, die diese Tätigkeit ausführen, überlassen $\gg{ }^{56}$ Wer ist damit gemeint? Spezialisten, wie man gewöhnlich annimmt - also die von Ärzten unterschiedenen Chirurgen oder, spezieller, Lithotomen? Solche Trennung der Chirurgie von der Medizin, solche Spezialisierung ist nach allem, was wir wissen, ein späthellenistisch-römisches Phänomen. Darf man es wagen, daraufhin den ganzen «Eid». entsprechend herunterzudatieren? Ich möchte lieber eine andere Interpretation 
des Satzes zur Diskussion stellen: Der «Eid»-Verfasser hat dasselbe sagen wollen wie der Autor von $\pi$. $\alpha \varrho \varrho \varrho \omega \nu 63$ («derartiges nicht einrenken, aber denjenigen von den Ärzten einrenken lassen, der will»). Doch wenn im «Eid» mit den «Männern» normale Ärzte gemeint sind - warum sind sie dann nicht (wie in

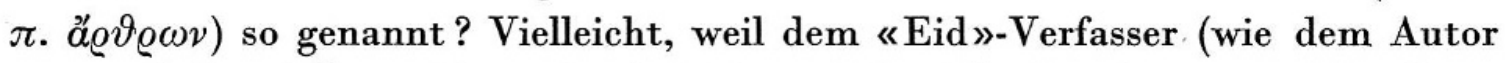
von $\pi . \not ้ \varrho \vartheta \varrho \omega \nu)$ Ärzte, die zu den kritisierten Eingriffen bereit waren, nicht einem hohen berufsethischen Anspruch genügten. Sie gehörten für ihn sicher in die

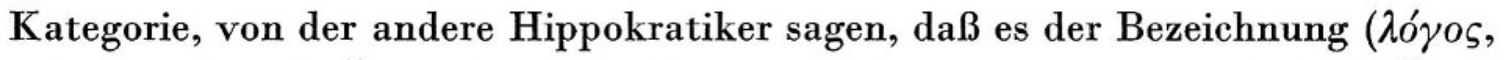

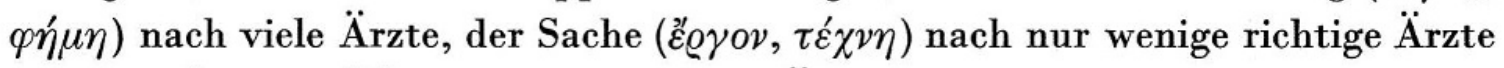

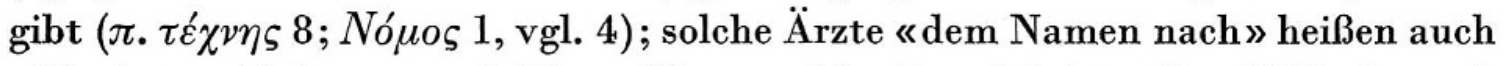

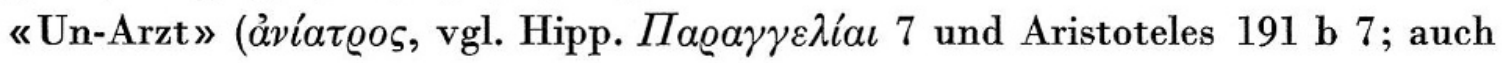

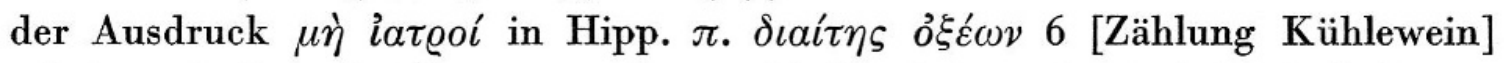
scheint mir diese Art Ärzte, von denen sich der Autor polemisch-kritisch distanziert, zu meinen $\left.{ }^{57}\right)$. Vielleicht hat der «Eid»-Verfasser deshalb, in hippokratischer Manier, durch eine entsprechende Umschreibung das «Un-Ärztliche» an den gemeinten Personen herausstellen wollen.

Hiermit spiegelt der «Eid» sicherlich keine «degradation of surgery » im Sinne der späteren Trennung von Chirurgie und Medizin ${ }^{58}$. Sondern es zeigt sich hier nicht der ganzen, sondern der operativen Chirurgie gegenüber - eine bei hippokratischen Internisten koischer Prägung vorgebildete, bei dem Hippokratiker Galen noch einmal scharf ausgeprägte Haltung, für die «in der überwiegenden Zahl chirurgischer Krankheitsfälle der operative Eingriff nicht einer echten Heilung gleichgesetzt, sondern zu einer dubiosen 'ultima ratio' entwertet » ist ${ }^{59}$. Daß das «... jedenfalls Steinleidende nicht» in hippokratischer medizinischer Anschauung gründet, sahen wir. Das generelle «ich werde nicht schneiden » mochte medizinisch, wie gesagt, der Haltung konservativer Internisten entsprechen. Als berufsethisches Gelübde aber unterwirft der ganze Passus die operative Chirurgie einer höchst strikten Auffassung des Gebots «nützen oder (doch) nicht schaden ».

Dies ist, im «Eid», ein deutliches Zeichen jenes eingangs angesprochenen berufsethischen Rigorismus. Hippokratiker, für die das Schneiden unproblematische Standardtherapie war (vgl. Aphorism. VII 87) - ebenso wie es dies seit je für das nichtärztliche Publikum war (vgl. Heraklit Vors. B 58 und Platon «Staat» 426 b) -, konnten wohl kaum dem schneidefeindlichen Gelübde des «Eides» beipflichten. Der «Eid» führte überdies mit seinem Rigorismus noch in ein besonderes, den Blasensteinschnitt betreffendes Dilemma: Wenn kein medikamentös bewirkter Steinabgang möglich war und die Operation unterblieb, dann litt (falls kein Schneidewilliger dawar) der Patient furchtbar, starb womöglich - Schädigung durch Unterlassung also für den «Eid»-Verpflichteten, und damit krasser 
Verstoß gegen das Gebot «nützen oder (doch) nicht schaden»! Ein späterer Hippokratiker zeigt, wie man in diesem Dilemma, berufsethisch abwägend, anders entscheiden kann: Laut Aretaios ist «ein großer Stein [in der Blase] unheilbar, denn er zerbröckelt nicht durch Trinken oder ein auflösendes Medikament. Geschnitten aber wird nicht ohne Schaden ..., und wenn auch der Patient der Todesgefahr entrinnt [nämlich durch Operation], folgt ihm chronischer Harnfluß; selbst falls das ungef ährlich ist, ist es für anständige Menschen nicht tragbar, daß der Urin immer fließt» (CMG II S. 69, 21-70, 1). Aretaios weiß also, anders als Hipp. Morb. I 8 (s.o.), daß manche Blasensteine nicht medikamentös zum Abgang zu bringen sind. Schneiden ist hier zwar für ihn nach wie vor ein Schädigen, kein Heilen. Dennoch: «Wenn kein Rat mehr für die Heilung ist und der Patient vor Schmerzen umkommt, in die Pubisregion und den Blasenhals schneiden ... und möglichst die Wunde zur Vernarbung bringen und heilen; falls aber nicht, dann ist es für das Weiterleben des Patienten besser, wenn chronischer Harnfluß entsteht, als wenn er durch Schmerz elendiglich umkommt» (a.a.O. S.139, 21-26). Das ist, im konkreten Fall, die Überwindung (durchaus in hippokratischem Geist!) eines zum absoluten Gebot geronnenen und daher ambivalenten berufsethischen Rigorismus im «Eid».

\section{Anmerkungen}

${ }^{1}$ Dazu s. H. Dillers «Forschungsgeschichtliches Nachwort» in: L. Edelstein, Der hippokratische Eid (übersetzt von K. Bartels), Zürich 1969, S. 97 f.

2 P. Herrmann (Hamburg) verdanke ich einige Hinweise auf epigraphischem Gebiet.

${ }^{3}$ Vgl. dazu zuletzt W. Burkert, Griechische Religion der archaischen und klassischen Epoche, Stuttgart 1977, S. 377-382.

${ }^{4}$ Vgl. E. Williger, Hagios. Untersuchungen zur Terminologie des Heiligen, Gießen 1922, S. 31 .

5 s. Burkert, a.a. O. S. $378 \mathrm{f}$.

6 Vgl. K. Deichgräber, Der hippokratische Eid, Stuttgart 1955, S. 27.

7 Vgl. Deichgräbers ältere (1933) Studie «Die ärztliche Standesethik des hippokratischen Eides », in: H. Flashar (Hrsg.), Antike Medizin, Darmstadt 1971, S. 117: «... das einzige (Gelöbnis), in dem ein religiöses Ethos fühlbar wird.»

8 Vgl. F.J. Dölger, Antike und Christentum 4, 1934 (Nachdr.1975), S.16ff. («der Hippokrates-Eid... läßt doch auch eine religiöse Motivierung vermuten»); L.Edelstein, Ancient medicine (hrsg. von O. und L. C. Temkin), Baltimore 1967, S. 15 («holiness ... is indicative of standards of ... a more elevated character», worunter Edelstein nicht «common religion», sondern Pythagoreisches versteht; ebd. Anm. 28 lehnt er O. Körners «ohne Fehl und unbescholten» ab als «a translation which in no way does justice to the meaning of the words»); Deichgräber, Der hippokratische Eid, a. a. O. («beide Ausdrücke entstammen dem Reinheitskult heiliger Gesetze, in denen die religiöse Befleckung der 
größte Schaden ist»; vgl. ebd. S. 14: der «Eid» gehöre «mitten hinein in die altgriechische Religion und Ethik»). Vgl. ferner E. L. Minar, Purity and holiness in the Hippocratic Oath, The Classical Weekly 40, 1947, S.151f. (nimmt mit Edelstein pythagoreischen Charakter für gegeben an).

9 Vgl. Edelstein, Ancient medicine, S. 326.

10 Vgl. Williger, a.a. O. S. 31 .

11 Ich bekenne gern, dazu gehört zu haben; vgl. Documenta Geigy: «Ärztliche Ethik», 1967, S.1, und Clio Medica 5 (1970), S.110.

12 J. Chr. Bolkestein, "O

13 In: Antike und Christentum 5 (1936, Nachdruck 1976), S. 282 f.

14 E. Benveniste, Indo-European language and society (Übersetzung von «Le vocabulaire des institutions indo-européennes "), London 1973, S. $465 \mathrm{ff}$.

15 ebd. S. 466.

16 ebd. S. 468 (unten).

17 Williger, a.a. O. S. 65-69.

18 ebd. S. 47 (unten).

19 Vgl. ebd. S. 66.

20 ebd. S. 66-68.

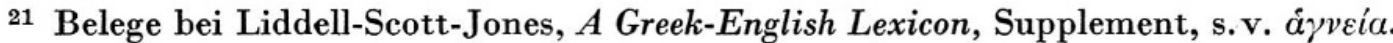

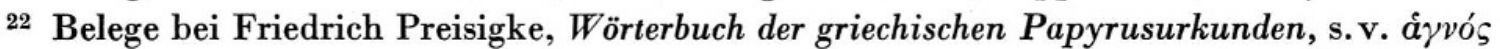
(«the expression was commonly applied to persons accepting office», Grenfell-Hunt zu Pap. Ox. 1413,11). Für Entsprechendes in Inschriften s. L. Robert in: Studii Clasice 16 (Bukarest 1974), S. 71 mit Anm. 8 (Robert übersetzt ebenfalls «intègre»).

${ }^{23}$ Vgl. Williger, a.a.0. S. $67 \mathrm{f}$.

${ }^{24}$ Belege bei Dittenberger, Orientis Graeci Inscriptiones Selectae, Bd. II, s.v. ó $\gamma v o ́ \varsigma$, und Williger, a.a.0. S. 67.

25 A. W.H.Adkins, Merit and responsibility, Oxford 1960, S.148 Anm. 2.

26 Benveniste, a.a.0. S. $461 \mathrm{ff}$.

27 ebd. S. 461.

28 ebd.

29 Vgl. K. J. Dover, Greek popular morality in the time of Plato and Aristotle, Oxford 1974, S. $252 \mathrm{f}$.

30 Dittenberger OGIS 339 Z.12 (2. Jahrhundert v. Chr.).

${ }^{31}$ Dittenberger, Syll. Inscr. Graec. ${ }^{3} 717$ Z. 88 (um 100 v. Chr.).

32 Vgl. A. Wilhelm in: Jahreshefte des Österreichischen Archäologischen Instituts 17 (Wien 1914), S. 36 (römische Zeit).

${ }^{33}$ M. van der Valk, Zum Worte ö $\sigma \iota \varsigma$, , in: Mnemosyne ${ }^{3} 10$ (1942), S.113-140.

${ }^{34}$ Dazu vgl. Dover, a.a. O. S.1-5.

35 ebd. S. 252.

${ }^{36}$ Dazu vgl. zuletzt M. P. Nilsson, Geschichte der griechischen Religion, Bd. 2, München ${ }^{2} 1961$, S. 290 f. (mit weiterer Literatur).

37 Zitiert bei Deichgräber, Ärztliche Standesethik, a.a.O. S.118 Anm. 54; s. auch Williger, a.a.O. S. 66.

38 Vgl. Kudlien, Clio Medica, a.a. O. S.107-111.

${ }^{39}$ Edelstein, Ancient medicine, S. 37 f., deutet auch dies in Richtung Pythagoreismus. 
${ }^{40} \mathrm{Vgl}$. dafür die paränetische Rede (Ps. Isokrates) «An Demonikos» § $22 \pi \varepsilon \varrho i \tau \tilde{\omega} v$

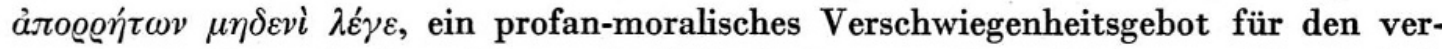
trauenswürdig/anständigen Mann. (Auf die Bedeutung dieser Rede zum Verständnis hippokratischer Berufsethik sei hier mit W. Müri, Griechische Studien, Basel 1976, S. 79 Anm.11, nochmals hingewiesen.)

41 Durch eine delphische Inschrift von ca. 360 v. Chr., hrsg. und erkl. von J. Bousquet in: Bulletin de la Correspondance Hellénique 80 (1956), S. 579-591, ist die Existenz der Asklepiaden als echter, profaner Familienverband (Zughörigkeit kraft Abstammung in männlicher Linie oder Adoption) dokumentarisch erwiesen. Damit erübrigen sich Spekulationen, daß das Lehrer-Schüler-Verhältnis im "covenant» des «Eides» eine "most sacred relationship» (Edelstein, Ancient medicine, S.40) sei, wie sie einer pythagoreischen Gemeinschaft (ebd. S.43 ff.) oder einem Mysterienkult bzw. der religiösen Ursprungsform einer Gilde (so Kudlien, Clio Medica, a.a.O. S.110) entspreche.

42 Dazu s. Edelstein, Ancient medicine, S. 26-30.

43 Vgl. seinen Übersetzungsband «Hippokrates. Schriften», Hamburg 1962, S. 9 Anm.

44 Ärtliche Standesethik, a.a. O. S. 99 (vgl. ebd. S. 109).

${ }^{45}$ In: Der hippokratische Eid, a. a. O. S. 39 (vgl. Text und Übersetzung ebd. S. 8 f.).

46 W. H. S. Jones, Hippocrates, vol. 1 (Loeb Classical Library), London 1923, S. 296.

47 Dazu vgl. W. Müri, Griechische Studien, a.a.O. S. 47-52.

48 Vgl. etwa Deichgräber, Ärztliche Standesethik, a.a.O. S. 99 Anm.11.

49 So S. Nittis, The Hippocratic Oath in reference to lithotomy, in: Bulletin of the History of Medicine 7 (1939), S.720.

50 Vgl. Deichgräber l.c. (oben Anm. 48).

51 J. D. Denniston, The Greek particles, Oxford 1966 (reprinted with corrections), S. 341.

52 Jones, a.a.O. S. 296 Anm. 2.

53 Denniston, a.a.O. S. 194.

54 ebd. S. 196.

55 ebd. S. 337.

${ }^{56}$ Der Übersetzungsversuch von Nittis (a.a.O. S.721): «and I will keep apart from men engaging in this deed", der die Kastrationstheorie stützen soll, ist schon vom Sprachlichen her abzulehnen (s. Edelstein, Ancient medicine, S. 27 Anm. 80).

${ }^{57}$ So faßt offenbar auch G. H. Knutzen, Technologie in den hippokratischen Schriften

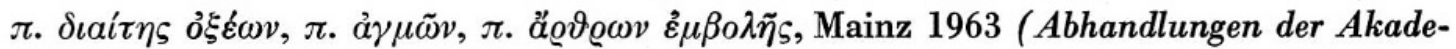
mie der Wissenschaften und der Literatur, geistes- und sozialwiss. Kl., Jg. 1963 Nr.14), S. 45 oben, den Ausdruck auf.

${ }^{58}$ So Jones, a.a. O. S. 296 Anm. 2.

59 Vgl. M. Michler, Das Spezialisierungsproblem in der antiken Chirurgie, Bern 1969, S. 58; s. auch insgesamt den Abschnitt «Die Rolle Galens», ebd. S. 50 ff. 


\section{Summary}

The author discusses his thoughts about two points of the Hippocratic Oath. 1. He tries to prove that the text is not a religious act, but a document of profane morals. 2 . The prohibition of lithotomy was not a general degradation of surgery, but a warning from a particular danger; the good medical doctor has to leave the operation to those who accept the risk.

Prof. Dr. phil. Fridolf Kudlien

Institut für Geschichte der Medizin und Pharmazie der Christian-Albrechts-Universität

D-2300 Kiel

Brunswiker Straße 2a 